$(\mathrm{n}=122)$ was measured in whole blood via LC-MS/MS. Data were analysed with a one-Way ANOVA.

Results We found statistically significant differences in maternal global DNA methylation $(\mathrm{p}=0.008)$ and global DNA hydroxymethylation $(\mathrm{p}=0.004)$ at 20 weeks of pregnancy. Post hoc tests revealed that global DNA methylation and global DNA hydroxymethylation level was significantly lower when the mother had an intellectual/scientific/artistic profession $(6.36 \%$ and $0.13 \%)$ as opposed to being a manager $(7.77 \%$, $\mathrm{p}=0.007 \%$ and $0.22 \%, \mathrm{p}=0.002)$ or administrative staff (7.71\%, $\mathrm{p}=0.003 \%$ and $0.2 \%, \mathrm{p}=0.005)$. No significant differences between different working groups were found for global DNA (hydroxy)methylation in the first and third trimester of pregnancy and at delivery.

Conclusion The mother's occupation was associated with maternal global DNA (hydroxy)methylation levels only in the second trimester of pregnancy. The change in maternal global DNA (hydroxy)methylation in the second trimester of pregnancy could be due to hormonal changes during pregnancy, a shift in the one-carbon metabolism in the middle of pregnancy, but based on these results we also have to take into account maternal occupational exposure.

\section{THE ASSOCIATION BETWEEN METAL CONCENTRATION IN HUMAN BODY AND SERUM ADVANCED GLYCATION END-PRODUCTS (AGES) AMONG METAL WORKERS}

\begin{abstract}
${ }^{1}$ Ying-Chuan Wang*, ${ }^{1}$ Chung-Ching Wang, 'Wei-Liang Chen, 'Gia-Chi Wang, 'TShengTa Chiang, ${ }^{1}$ Fang-Yih Liaw, ${ }^{2}$ Wei-Te Wu, 1,2,3 Saou-Hsing Liou*. 'Division of Occupational Medicine, Department of Family and Community Medicine, Tri-Service General Hospital, National Defense Medical Centre, Taipei, Taiwan, Republic of China; ${ }^{2}$ National Institute of Environmental Health Science, National Health Research Institutes, Miaoli, Taiwan, Republic of China; ${ }^{3}$ Department of Public Health, National Defense Medical Centre, Taipei, Taiwan, Republic of China
\end{abstract}

\subsection{6/oemed-2018-ICOHabstracts.385}

Introduction Advanced glycation end-products (AGEs) are the products formed by physical adhesion of serum glucose to some serum macromolecules such as proteins or nucleotides after a series of chemical reactions. Based on past studies, AGEs are associated with many diseases, especially cardiovascular diseases such as T2DM and atherosclerosis. Therefore, we conducted a study to explore the association between metal concentration in human body and serum AGEs.

Methods This study is a cross-sectional design, and its study population is composed of 283 workers exposed to metal in northern Taiwan. We used questionnaires to obtain the basic demographic data of the workers. Also, we collected the blood and urine samples from the workers, and we used appropriate methods to analyse the serum and urine samples including the concentration of metal, markers about metabolic diseases and serum advanced glycation end-products. We performed statistical methods containing mainly multivariable regression to analyse the data.

Result According to the study results, we found the serum nickel $(\mathrm{Ni})$, serum lead $(\mathrm{Pb})$, and serum thallium $(\mathrm{Tl})$ are statistically crucial to the dependent variable serum AGEs concentration even after adjusting the possible covariates comprising age, gender, $\mathrm{HbA} 1 \mathrm{C}$, total cholesterol, BMI, diet habits, the smoking habit, medical history of hypertension, medical history of coronary artery disease and medical history of chronic obstructive pulmonary disease. The regression coefficients $\mathrm{B}$ for $\mathrm{Ni}, \mathrm{Pb}$, and $\mathrm{Tl}$ are 0.15 (95\% CI: 0.06 0.24; p<0.001),
0.17 (95\% CI: $0.10 \sim 0.24 ; \mathrm{p}<0.001)$, and 0.20 (95\% CI: $0.02 \sim 0.38 ; \mathrm{p}<0.05)$ respectively. Besides, we acquired significant positive associations between serum AGEs concentration and any of the Framingham risk score.

Discussion According to our research findings, we suggested the positive association between serum $\mathrm{Ni}, \mathrm{Pb}$ and $\mathrm{Tl}$ levels with serum AGEs concentration. Furthermore, the workers with higher serum $\mathrm{Ni}, \mathrm{Pb}$ and $\mathrm{Tl}$ levels might increase 10 year risk of cardiovascular disease via the pathogenic processes of AGEs.

\section{COPENHAGEN PSYCHOSOCIAL QUESTIONNAIRE-3 (COPSOQ-3): TURKISH VALIDATION STUDY}

${ }^{1}$ Ceyda Sahan, ${ }^{2}$ Hakan Baydur, 1,3Yucel Demiral. 'Occupational Medicine Department, Dokuz Eylul University, Izmir, Turkey; ${ }^{2}$ Department of Social Work, School of Health Sciences, Celal Bayar University, Manisa, Turkey; ${ }^{3}$ Public Health Department, Dokuz Eylul University, Izmir, Turkey

\subsection{6/oemed-2018-ICOHabstracts.386}

Introduction The Copenhagen Psychosocial Questionnaire-3 (COPSOQ-3), has been developed to assess psychosocial risks in the workplace. questionnaire consisted 25 dimensions as follows: work pace, quantitative demands, emotional demands, demands for hiding emotions (DHE), influence at work, possibilities for development, degree of freedom at work (DFW), meaning of work, commitment to the workplace, predictability, recognition, role-clarity, role-conflicts, quality of leadership, social support from colleagues, social support from supervisors, sense of community, insecurity over employment, insecurity over working conditions, work-life conflict, trust, organisational justice and job satisfaction. In total, COPSOQ3 -TR version included 78 items and 23 dimensions. The aim of the study was validation of the form of the COPSOQ-3 to Turkish language.

Methods This is a methodologic study. The field study has been carried out in three workplaces (Call centre, plastic and metal industry) Kaiser-Meyer-Olkin (KMO) Measure of Sampling Adequacy and Bartlett's Test of Sphericity were used to assess the suitability of the sample for factor analysis. Validity of the dimensions were investigated with exploratory factor analysis. The principal component analysis and varimax rotation methods used to identify the factor structure. The internal consistency was assessed by the Cronbach's alpha coefficient.

Results 807 respondents' questionnaires were evaluated. Fiftyseven percent of them were men. Mean age of men and women were $32.1 \pm 7.4,27.1 \pm 6.6$, respectively.Sampling adequacy was considered adequate $(\mathrm{KMO}=0.884)$. The factor analysis of COPSOQ-TR identified seventeen factors with eigenvalue higher than one and explained\%63.6 of the total variance.The Cronbach's alpha values of 20 dimensions were found between 0.705 and 0.884 . The Cronbach's alpha values of two dimensions (DHE and DFW) were found 0.638 and 0.643. The model is found that excellent fit (ChiSquare $=5860.80, \mathrm{df}=2672, \mathrm{P}$-value $=0.00000, \mathrm{RMSEA}=0.038$, $\mathrm{SRMR}=0.05, \mathrm{CFI}=0.97)$.

Discussion Findings have shown that Turkish version of the Copenhagen Psychosocial Questionnaire-3 is a reliable and valid instrument that can be considered useful tool to measure the psychosocial risks in Turkish population. 